\title{
CYP2D6 Genotyping with Oligonucleotide Microarrays and Nortriptyline Concentrations in Geriatric Depression
}

Greer M. Murphy, Jr., M.D., Ph.D., Bruce G. Pollock, M.D., Ph.D., Margaret A. Kirshner, B.A., Nina Pascoe, B.S., William Cheuk, B.A., Benoit H. Mulsant, M.D., and Charles F. Reynolds III, M.D.

Recent advances in oligonucleotide microarray technology ("gene chips") permit rapid screening for DNA sequence variation. The CYP2D6 gene encodes debrisoquine hydroxylase, which metabolizes the antidepressant nortriptyline and other psychotropic medications. Nortriptyline plasma concentrations were obtained after at least three weeks of treatment in 36 geriatric patients with major depression who were taking a mean of 8.6 other medications besides nortriptyline. Oligonucleotide microarrays were used to detect 16 CYP2D 6 alleles that affect debrisoquine hydroxylase activity. Subjects carrying alleles encoding impaired debrisoquine hydroxylase activity had significantly greater nortriptyline concentrations and lower nortriptyline doses than did other subjects. Significant correlations were found between the numbers of alleles encoding decreased metabolism and nortriptyline plasma concentration, nortriptyline dose, and nortriptyline plasma concentration standardized for dose, indicating a gene dosage effect. These results demonstrate that CYP2D6 genotyping on a microarray platform can be used to predict plasma antidepressant concentrations despite advanced patient age and numerous concurrent medications.

[Neuropsychopharmacology 25:737-743, 2001] (C) 2001 American College of Neuropsychopharmacology. Published by Elsevier Science Inc.
KEY WORDS: Antidepressants; CYP2D6; Oligonucleotide array sequence analysis; Nortriptyline; Depressive disorder; Geriatric psychiatry

From the Department of Psychiatry and Behavioral Sciences, Stanford University School of Medicine, Stanford, CA (GMM, NP, WC); Department of Veterans Affairs Sierra-Pacific Mental Illness Research, Education, and Clinical Center, Palo Alto, CA (GMM, $\mathrm{NP}$ ); Intervention Research Center for Late-Life Mood Disorders, University of Pittsburgh Medical Center, Pittsburgh, PA (BGP, MAK, BHM, CFR)

Address correspondence to: Greer M. Murphy, Jr., M.D., Ph.D., Neuroscience Research Laboratories, Department of Psychiatry and Behavioral Sciences, MSLS P-104, Stanford University School of Medicine, Stanford, CA 94305-5485, Tel.: 650-725-0565, Fax: 650-7255714 or 650-498-7761, e-mail: gmurphy@leland.stanford.edu

Received February 13, 2001; revised April 24, 2001; accepted May 3, 2001.

Online publication: 5/15/01 at www.acnp.org/citations/Npp 051501120 .
Oligonucleotide microarrays, also called "gene chips", represent a dramatic minaturization of several important molecular biology techniques (Watson and Akil 1999). Because of the small size of each array element, many reactions can be performed simultaneously, resulting in significant savings in time and labor. The most widespread use of oligonucleotide microarrays at present is for analysis of gene expression (Fodor 1997; Alon et al. 1999; Lee et al. 1999). In that application, messenger RNA levels for tens of thousands of genes are determined simultaneously by hybridizing RNA or a derivative from cell or tissue samples with the array. However, oligonucleotide microarrays may also be used to detect polymorphisms in DNA (Chee et al. 1996; Cargill et al. 1999). Typically genomic DNA is amplified, labeled, and hybridized with a microarray containing thousands of DNA oligonucleotides corresponding to allelic DNA sequences. Hundreds or thousands of 
DNA polymorphisms can be detected on a single array by analyzing the binding of the target DNA with the oligonucleotides.

The tricyclic antidepressant nortriptyline shows a favorable side effect profile in comparison with most other tricyclic agents (Potter et al. 1998). Plasma nortriptyline concentrations between 50 and $150 \mathrm{ng} / \mathrm{ml}$ are associated with antidepressant efficacy, whereas higher concentrations result in side effects and loss of efficacy (Perry et al. 1994; Burke and Preskorn 1999). Debrisoquine hydroxylase, encoded by the CYP2D6 gene, is the principal enzyme for the oxidative metabolism of nortriptyline (Olesen and Linnet 1997). Certain CYP2D6 mutations impair or abolish debrisoquine hydroxylase activity, resulting in reduced metabolism of nortriptyline and high plasma nortriptyline concentations even at low doses (Bertilsson et al. 1997). CYP2D6 genotyping has been proposed as a clinical laboratory method for predicting plasma concentrations of nortriptyline and other antidepressant medications (Linder et al. 1997). However, there are numerous CYP2D6 mutations that affect drug metabolism, and genotyping using conventional methods such as restriction isotyping is labor intensive involving multiple polymerase chain reactions, restriction enzyme digestions, and separations of digested DNA fragments by gel electrophoresis (Sachse et al. 1997). Further, all prior studies of CYP2D6 genotype and nortriptyline levels have utilized single dose pharmacokinetic designs or clinical samples of predominantly younger patients taking few concurrent medications (Dahl et al. 1996; Dalen et al. 1998; Yue et al. 1998; Morita et al. 2000). Results from these prior studies may not be applicable to geriatric patients prescribed nortriptyline in clinical settings where large numbers of concurrent medications are also utilized that may alter debrisoquine hydroxylase activity.

We used oligonucleotide microarrays to genotype depressed geriatric patients for mutations at the CYP2D6 locus that affect debrisoquine hydroxylase activity and hence nortriptyline metabolism. The patients were taking a mean of 8.6 other medications at the time nortriptyline concentrations were determined. Geriatric patients are particularly vulnerable to nortriptyline side effects at high plasma concentrations (Pollock et al. 1994; Pollock 1999). Our results indicate that CYP2D6 genotyping with oligonucleotide microarrays is an effective means of predicting nortriptyline levels in geriatric patients despite concurrent therapy with a wide variety of other medications.

\section{MATERIALS AND METHODS}

Subjects were 36 patients recruited from the geriatric inpatient units and the outpatient Late Life Depression Clinic of Western Psychiatric Institute and Clinic. 32 of the subjects were Caucasian, three were African-American, and one was Asian-American. There were $21 \mathrm{fe}-$ males and 15 males. The mean age was 73.1 years (range 60 to 95 years of age). For inclusion in the study, patients had to meet the following criteria: age 60 years or older; DSM-IV major depressive episode without psychotic features or history of bipolar or schizoaffective disorder; baseline HRSD score of 15 or above; MMSE score of 18 or above; no history of alcohol or other substance abuse or dependency during at least the past year; and no specific medical condition contraindicating treatment with nortriptyline (e.g., QRS longer than $120 \mathrm{~ms}$ ). All protocols had received Institutional Review Board approval, and all patients provided written informed consent. Nortriptyline was started at $25 \mathrm{mg}$ per day for outpatients and at $50 \mathrm{mg}$ per day for inpatients. Dose was adjusted as needed to obtain a plasma nortriptyline concentration between 50 and $150 \mathrm{ng} / \mathrm{ml}$. Prescribing physicians were unaware of the results of CYP2D6 genotyping.

Plasma nortriptyline concentrations were measured by high performance liquid chromatography with ultraviolet detection. The assay was linear from 10 to 300 $\mathrm{ng} / \mathrm{ml}$ with inter-assay coefficients of variation ranging from 2.4 to $3.5 \%$ (Pollock et al. 1992). All nortriptyline concentrations used for correlation with genetic data were obtained after at least three weeks at a given dose. During the study period patients remained on other prescription or over the counter medications that they had been taking before the initiation of nortriptyline therapy. On the average, subjects were taking 8.6 (S.E. = 0.79) concurrent medications in addition to nortriptyline at the time nortriptyline concentrations were determined. For the entire sample of 36 patients, a total of 121 unique medications were used concurrently during the study period including antibiotics, antihypertensives, antianginals, vitamins and minerals, diuretics, oral hypoglycemics, benzodiazepines, and others. One of the subjects took a medication that is known to inhibit CYP2D6 during the study period (cimetidine).

Leukocytes were obtained from whole blood using LeukoPrep tubes or CPT Vacutainer tubes (Becton Dickinson, Franklin Lakes, NJ). Genomic DNA was obtained from leukocyte pellets with the QIAamp Blood Mini Kit according to the manufacturer's instructions (Qiagen, Valencia, CA) or by a standard proteinase $\mathrm{K}$ digestion and salting-out procedure that included lysing cells in a 10:2 mixture of Tris-EDTA buffer (10mM Tris $\mathrm{HCl}, 0.2 \mathrm{mM}$ EDTA) and $5 \mathrm{M} \mathrm{NaCl}$ followed by $10 \%$ SDS treatment, proteinase $\mathrm{K}$ digestion, removal of cellular debris by centrifugation, precipitation of DNA with $5 \mathrm{M} \mathrm{NaCl}$ and $100 \%$ ethanol, washing with $70 \%$ ethanol, and resuspension of DNA in Tris-EDTA buffer. CYP2D6 genotyping was performed using the GeneChip CYP450 assay (Affymetrix, Santa Clara, CA) following the manufacturer's in- 
structions exactly (Affymetrix GeneChip CYP450 assay manual). Briefly, five multiplex polymerase chain reactions (PCR) were used to amplify exons 1 through 9 of the CYP2D6 gene from genomic DNA samples. Amplicons were fragmented using a proprietary reagent (Affymetrix), treated with alkaline phosphatase, and 3' labeled with fluoresceinated-N6-dideoxyATP using terminal transferase. Labeled samples were reacted with GeneChip CYP450 probe arrays in an Affymetrix GeneChip Fluidics Station, and then arrays were laser scanned in a GeneArray Scanner (Hewlett Packard, Palo Alto, CA). Based on the hybridization pattern of the labeled sample to the 8000 element array (2920 of which interrogate CYP2D6 sequences), the presence or absence of 16 allelic variants of the CYP2D6 gene was determined. To detect CYP2D6 gene deletions, the method of Steen et al. (Steen et al. 1995) was used. A positive control template was included with every long-range PCR amplification used to detect gene deletions.

The nomenclature system of Daly et al. (1996) was used for CYP2D6 alleles. We classified the most common CYP2D6 alleles according to predicted metabolic activity: extensive $\left({ }^{*} 1\right)$, intermediate $\left({ }^{*} 2,{ }^{*} 10\right)$, or null $\left({ }^{*} 3\right.$, $\left.{ }^{*} 4 \mathrm{~A},{ }^{*} 5\right)$ based on published genotype-phenotype comparisons (Marez et al. 1997; Sachse et al. 1997). Subjects were classified as having a genotype resulting in extensive drug metabolism (two extensive metabolic alleles or an extensive metabolic allele and an intermediate metabolic allele), or a genotype resulting in impaired drug metabolism (two intermediate metabolic alleles, one null allele, or two null alleles). Although subjects with two null alleles have greater impairment of debrisoquine hydroxylase activity than subjects with two intermediate metabolic alleles or one null allele (Sachse et al. 1997), the small number of homozygous null genotypes in our sample precluded analysis of these subjects separately. Mean nortriptyline concentrations, nortriptyline doses, and nortripyline concentrations per unit dose were compared between the groups using two-tailed t-tests. Subjects were also assigned a mutation score based on the number of extensive, intermediate, and null alleles they carried. Extensive metabolic alleles were scored as 0 , intermediate metabolic alleles as 0.5 , and null alleles as 1.0. Thus, because each subject carried two alleles, this mutation score ranged from 0 to 2.0. Spearman Rho correlation coefficients were calculated between mutation scores and nortriptyline concentrations, nortriptyline doses, and nortriptyline concentrations per unit dose.

To test the accuracy of the microarray assay, we compared microarray results from nine additional samples not included in the clinical study to results obtained using a conventional PCR, restriction enzyme, and gel electrophoresis genotyping system (Sachse et al. 1997).
Table 1. Allele Frequencies

\begin{tabular}{lcccccc}
\hline Allele & ${ }^{*} \mathbf{1}$ & ${ }^{*} \mathbf{2}$ & ${ }^{*} \mathbf{3}$ & ${ }^{*} \mathbf{4 A}$ & ${ }^{*} \mathbf{5}$ & ${ }^{*} \mathbf{1 0 B}$ \\
\hline Frequency & 0.41 & 0.32 & 0.04 & 0.15 & 0.01 & 0.07 \\
\hline
\end{tabular}

\section{RESULTS}

In the sample of 36 subjects we detected six different CYP2D6 alleles (Table 1). Allele frequencies were similar to those found in large sample studies (Marez et al. 1997; Sachse et al. 1997). Among the 36 subjects there were 11 genotypes, which are listed in Table 2. Eighteen subjects had genotypes giving rise to extensive metabolism $\left({ }^{*} 1 /\right.$ ${ }^{*} 2,{ }^{*} 1 /{ }^{*} 10 \mathrm{~B},{ }^{*} 1 /{ }^{*} 1$ ), whereas 18 subjects had genotypes giving rise to impaired metabolism $\left({ }^{*} 1 /{ }^{*} 4 \mathrm{~A},{ }^{*} 2 /{ }^{*} 4 \mathrm{~A},{ }^{*} 2 /\right.$ ${ }^{*} 10 \mathrm{~B},{ }^{*} 1 /{ }^{*} 3, * 2 /{ }^{*} 2,{ }^{*} 10 \mathrm{~B} /{ }^{*},{ }^{*} 3 /{ }^{*} 4 \mathrm{~A},{ }^{*} 4 \mathrm{~A} /{ }^{*} 4 \mathrm{~A}$ ).

Subjects with genotypes giving rise to impaired metabolism had significantly higher mean nortriptyline concentrations $(\mathrm{t}=2.5$, d.f. $=34, p<.02$; Table 3$)$ and significantly higher nortriptyline concentrations per unit dose of the medication $(\mathrm{t}=4.9$, d.f. $=34, p<.0001$; Table 3 and Figure 1) than did those with genotypes associated with wild-type metabolism. Nortriptyline doses in subjects with genotypes encoding impaired metabolism were significantly lower than in other subjects $(t=3.4$, d.f. $=34, p<.002$; Table 3$)$. There were significant correlations between mutation score and nortriptyline concentration (rho $=0.46, p<.005$ ), nortriptyline dose (rho $=-0.58, p<.001$ ) and nortriptyline concentration per unit dose (rho $=0.73, p<$ .0001; Figure 2).

Because the nortriptyline metabolic phenotype associated with intermediate metabolic alleles has not been definitively established, we performed the same analyses with subjects who had two intermediate metabolic alleles $(n=5)$ classified with subjects having genotypes encoding extensive metabolism. This did not change the outcome, with the 13 subjects carrying one or more

Table 2. Genotype Frequencies

\begin{tabular}{|c|c|c|c|}
\hline Genotype & Frequency & Percent & Cumulative Percent \\
\hline$* 1, * 1$ & 5 & 13.9 & 13.9 \\
\hline${ }^{*} 1,{ }^{*} 10 \mathrm{~B}$ & 1 & 2.8 & 16.7 \\
\hline${ }^{*} 1,{ }^{*} 2$ & 12 & 33.3 & 50.0 \\
\hline$* 1, * 3^{\ddagger}$ & 2 & 5.6 & 55.6 \\
\hline$* 1, * 4 A^{\ddagger}$ & 4 & 11.1 & 66.7 \\
\hline${ }^{*} 10 \mathrm{~B},{ }^{*} 5^{\ddagger}$ & 1 & 2.8 & 69.4 \\
\hline$* 2, * 10 \mathrm{~B}^{\ddagger}$ & 3 & 8.3 & 77.8 \\
\hline$* 2, * 2 \ddagger$ & 2 & 5.6 & 83.3 \\
\hline$* 2, * 4 A^{\ddagger}$ & 4 & 11.1 & 94.4 \\
\hline$* 3, * 4 \mathrm{~A}^{\#}$ & 1 & 2.8 & 97.2 \\
\hline${ }^{*} 4 \mathrm{~A},{ }^{*} 4 \mathrm{~A}^{\#}$ & 1 & 2.8 & 100.0 \\
\hline Total & 36 & 100.0 & \\
\hline
\end{tabular}

$\ddagger$ Intermediate metabolism genotype

\# Poor metabolism genotype 
Table 3. Means and Standard Errors

\begin{tabular}{lll}
\hline & \multicolumn{1}{c}{ No mutation $(\mathbf{N}=\mathbf{1 8})$} & \multicolumn{1}{c}{ Mutation $(\mathbf{N}=\mathbf{1 8})$} \\
\hline Nortriptyline Concentration & Mean $=79.3 \mathrm{ng} / \mathrm{ml}($ S.E. $=5.3)$ & Mean $=115.1 \mathrm{ng} / \mathrm{ml}($ S.E. $=13.1)$ \\
Nortriptyline Dose & Mean $=66.9 \mathrm{mg}($ S.E. $=6.1)$ & Mean $=43.3 \mathrm{mg}(\mathrm{S} . E .=3.2)$ \\
Nortriptyline Concentration per Unit Dose & Mean $=1.3 \mathrm{ng} / \mathrm{ml}($ S.E. $=0.1)$ & Mean $=2.9 \mathrm{ng} / \mathrm{ml}($ S.E. $=0.3)$ \\
\hline
\end{tabular}

null alleles having significantly higher nortriptyline levels per unit dose than the other 23 subjects $(t=2.9$, d.f. $=34, p<.006)$.

Nine additional DNA samples (genotypes ${ }^{*} 1 /{ }^{*} 1,{ }^{*} 1 /$ ${ }^{*} 10 \mathrm{~B},{ }^{*} 4 \mathrm{~A} /{ }^{*} 4 \mathrm{~A},{ }^{*} 2 /{ }^{*} 10 \mathrm{~B},{ }^{*} 1 /{ }^{*} 10 \mathrm{~B},{ }^{*} 2 /{ }^{*} 10 \mathrm{~B},{ }^{*} 1 /{ }^{*} 10 \mathrm{~B}$, $\left.{ }^{*} 6 \mathrm{~B} /{ }^{*} 10 \mathrm{~B},{ }^{*} 2 /{ }^{*} 10 \mathrm{~B}\right)$ were evaluated with both the microarray system and with conventional CYP2D6 genotyping (Sachse et al. 1997). In each case the results were identical.

\section{DISCUSSION}

These results demonstrate that geriatric patients identified by CYP2D6 microarray genotyping as having impaired drug metabolism have significantly higher nortriptyline concentrations than those with genotypes associated with extensive metabolism. This difference was particularly striking when nortriptyline concentrations were normalized for dosage. Patients with genotypes for impaired metabolism had a mean nortriptyline concentration per unit dose that was over twice as high as those with genotypes encoding extensive metabolism. Further, nortriptyline doses were on the average about one-third lower in patients with genotypes encoding impaired metabolism. There were significant correlations between the number of intermediate and null alleles (mutation score) and nortriptyline concentration, nortriptyline dose, and nortripyline concentration per unit dose. These findings indicate that CYP2D6 genotyping using oligonucleotide microarrays is an effective means of identifying those patients who will have high nortriptyline concentrations at standard doses.

There was a strong association between CYP2D6 genotype and plasma nortriptyline concentrations despite the advanced age of the patients and concurrent use of an average of 8.6 prescription and over-the-counter medications besides nortriptyline. There has been concern that the correlation between CYP2D6 genotype and metabolic phenotype might change with age (Pollock et al. 1994; Mulsant et al. 1997), and that concurrent use of large numbers of medications in the elderly could alter metabolic phenotype so as to mask the effects of genotype (Coutts 1994). All prior studies of CYP2D6 genotype and nortriptyline metabolism have been single dose pharmacokinetic studies that may have limited application to clinical practice, or have in- volved largely younger subjects. Dahl et al. (1996) studied 21 caucasian depressed patients younger than 65 years of age treated with a fixed dose of $150 \mathrm{mg}$ nortriptyline per day. The number of concurrent medications was not specified. They found that genotype (only ${ }^{*} 1$, $* 3, * 4$, and $* 5$ were examined) predicted nortriptyline level, but only when gender was included in the regression model. Indeed, gender was a stronger predictor of nortriptyline concentration than was CYP2D6 genotype. Dalen et al. (1998) gave 21 healthy Caucasian subjects, ages 18 to 48, single doses of nortriptyline (other medications unspecified) and measured pharmacokinetics over the next 168 hours. They found significant corrrelations between CYP2D6 genotype and nortriptyline clearance. Similarly, Yue et al. (1998) gave single nortriptyline doses to 15 healthy Chinese subjects ages 22 to 46 (no concurrent medications) and measured nortriptyline pharmacokinetics for 168 hours. They found that among Chinese the ${ }^{*} 10$ allele was associated with higher nortriptyline levels than was the ${ }^{*} 1$ allele. Finally, Morita et al. (2000) examined 41 Japanese pa-

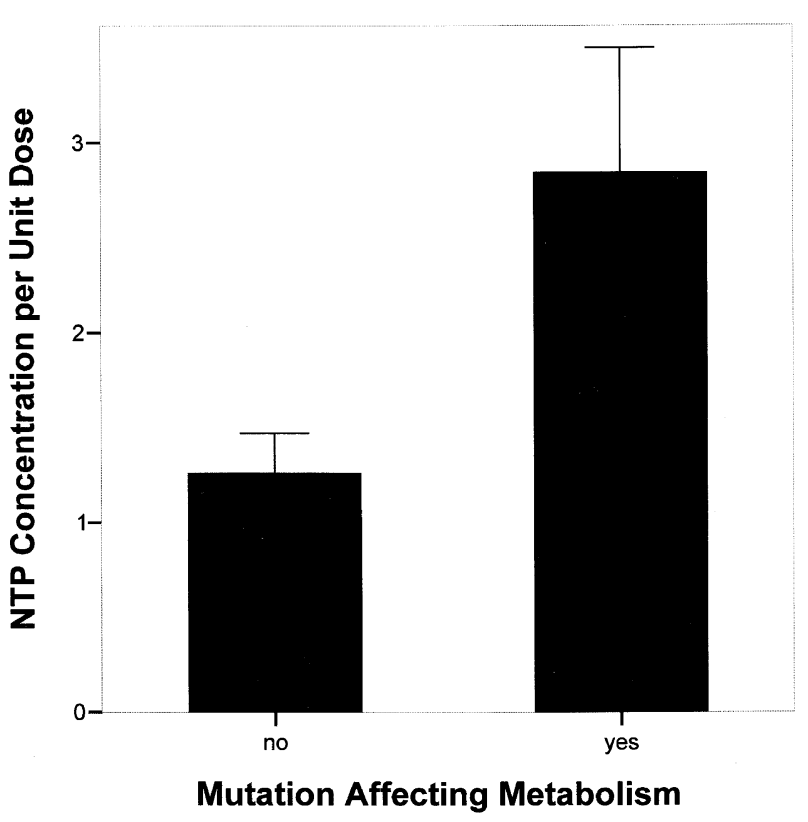

Figure 1. Patients with genotypes associated with decreased debrisoquine hydroxylase activity have increased plasma nortriptyline concentrations per unit dose. Bars represent mean nortriptyline concentration per unit dose with standard errors. 


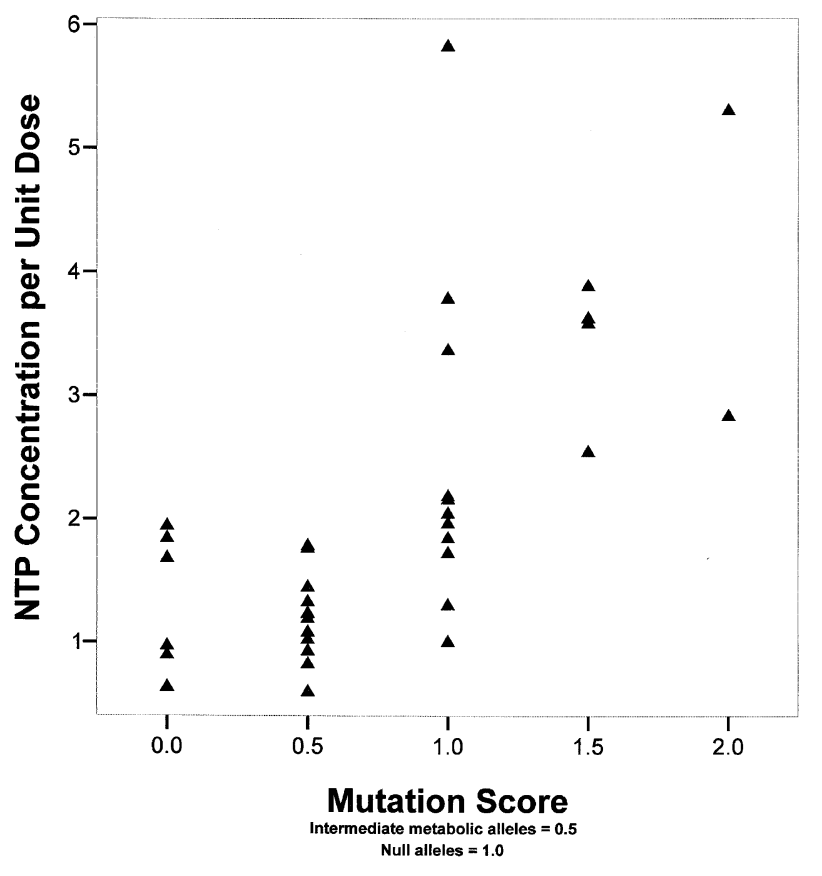

Figure 2. CYP2D6 mutation score is correlated with nortriptyline concentration per unit dose. Patients were assigned a mutation score based on the number of extensive, intermediate, and null CYP2D6 alleles they carried. Extensive metabolic alleles were scored as 0 , intermediate metabolic alleles as 0.5 , and null alleles as 1.0. Thus, because each subject carried two alleles, this mutation score ranged from 0 to 2.0. This score is plotted on the $\mathrm{x}$-axis with nortriptyline concentration per unit dose plotted on the y-axis. A correlation between the two measures is evident.

tients ages 17 to 76 (mean 48.6) years of age with a variety of psychiatric diagnoses who were taking no other medications except benzodiazepines. They found significant differences in nortriptyline levels between subjects with differing numbers of CYP2D6 alleles encoding decreased metabolism.

None of the prior studies of CYP2D6 genotype and nortriptyline metabolism address the issue of the predictive value of CYP2D6 genotype in old age, and none address the issue of whether CYP2D6 genotype is penetrant in the setting of a large number of concurrent medications. Our results show that CYP2D6 genotyping predicts nortriptyline levels as well in geriatric patients taking numerous medications as it does in younger patients (Dahl et al. 1996). CYP2D6 genotype effects on antidepressant drug concentrations were not masked by interactions between the desbrisoquine hydroxylase and the large number of concurrent medications typically prescribed for geriatric patients. Further, the correlation detected between CYP2D6 genotype and nortriptyline levels in our sample was similar to that between debrisoquine phenotype and nortriptyline levels reported by others. It is noteworthy that a gene dosage effect on nortriptyline levels per unit dose is apparent even in this relatively small sample. However, our data do not address the issue of whether CYP2D6 genotype predicts nortriptyline metabolism in patients prescribed a strong inhibitor of CYP2D6 during nortriptyline therapy (Pollock et al. 1994; Mulsant et al. 1997).

Oligonucleotide microarrays represent a significant advance over older genotyping techniques. The microarray platform utilized in the present study identifies sixteen common CYP2D6 alleles. Starting with genomic DNA samples, the entire process can be completed in one day, and multiple samples can be analyzed simultaneously. In contrast, genotyping for the same alleles using PCR followed by restriction digestion requires 12 different PCR reactions followed by 11 different restriction digestions and 23 lanes of gel electrophoresis for each subject (Sachse et al. 1997) assuming that every reaction is successful on the first attempt. Debrisoquine phenotyping can also be difficult. Patients must ingest debrisoquine or other enzyme substrate, followed by collection of one or more urine samples hours later, which are then subjected to gas chromatography or other biochemical analysis (Coutts 1994). Further, whereas debrisoquine phenotype values may be affected by concurrent medications that inhibit debrisoquine hydroxylase, CYP2D6 genotype is fixed at birth and invariant.

In our hands the total cost of materials and labor per microarray was approximately $\$ 200$. This amounts to about $\$ 12$ per polymorphism determined by a single array. One half-time technician can readily process 50 arrays per week, or 850 polymorphisms. It should be noted that in the present study we performed an additional genotyping assay for CYP2D6 gene deletions $\left({ }^{*} 5\right.$ allele) that was not included on the array. Further, the array does not detect CYP2D6 gene duplications that occur in approximately 3\% of caucasians (Sachse et al. 1997). Performing this assay might increase the accuracy of prediction.

The microarrays used in the present study are of low density, consisting of approximately 8,000 array elements. Each element contains multiple copies of oligonucleotides that probe for single nucleotide polymorphisms and other sequence variants in patient DNA samples. Microarrays of far higher density are feasible using currently available technology. A "custom" array designed to identify polymorphisms in other candidate pharmacogenes for antidepressant side effects and/or efficacy could be devised and implemented immediately using a new generic microarray format (Hirschhorn et al. 2000). This could expedite the screening of large numbers of subjects treated with antidepressants for genetic determinants of medication efficacy and tolerance.

Nortriptyline has one of the best side effect profiles of all tricyclic antidepressant medications (Potter et al. 
1998). The efficacy of nortriptyline as an antidepressant has been demonstrated in numerous clinical trials. Further, among all antidepressant medications, nortriptyline concentrations probably have the closest correlation with clinical efficacy and with side effects (Perry et al. 1994; Burke and Preskorn 1999). However, many clinicians currently avoid prescribing this medication because of concerns about side effects. By using CYP2D6 genotyping, clinicians should be able to identify patients with mutant alleles who are poor candidates for nortriptyline therapy, as well as those likely to be good candidates for this medication. This may be particularly important in light of recent evidence that nortriptyline efficacy is greatest at higher serum concentrations where the margin of safety is smallest (Reynolds et al. 1999). Potentially, CYP2D6 genotyping with microarrays could allow physicians to choose starting doses based on predicted metabolic impairment. This might decrease the frequencies of early side effects, plasma drug level monitoring, and dosage adjustments. It is recognized that overall use of nortriptyline is decreasing, but our results should still be applicable to selected patients for whom this agent is particularly indicated (Robinson et al. 2000).

In summary, CYP2D6 genotyping with oligonucleotide microarrays can identify subjects who may experience high nortriptyline plasma concentrations at standard dosages. To our knowledge this is the first application of this rapidly developing genetic technology to an important problem in clinical psychiatry. In order to fully assess its clinical utility, future studies should determine if oligonucleotide microarray genotyping can predict actual clinical outcome in patients treated with nortriptyline and other CYP2D6 substrates.

\section{ACKNOWLEDGMENTS}

Supported by grants from the Nancy Pritzker Network for the Study of Depression, the National Institute of Mental Health (MH01239, MH01509, MH52247, MH55106, MH30915, and MH40041), The National Alliance for Research on Schizophrenia and Depression, and the Department of Veterans Affairs (Sierra-Pacific Mental Illness Research, Education, and Clinical Center).

\section{REFERENCES}

Alon U, Barkai N, Notterman DA, Gish K, Ybarra S, Mack D, Levine AJ (1999): Broad patterns of gene expression revealed by clustering analysis of tumor and normal colon tissues probed by oligonucleotide arrays. Proc Natl Acad Sci USA 96:6745-6750

Bertilsson L, Dahl ML, Tybring G (1997): Pharmacogenetics of antidepressants: clinical aspects. Acta Psychiatr Scand Suppl 391:14-21
Burke MJ, Preskorn SH (1999): Therapeutic drug monitoring of antidepressants: cost implications and relevance to clinical practice. Clin Pharmacokinet 37:147-165

Cargill M, Altshuler D, Ireland J, Sklar P, Ardlie K, Patil N, Shaw N, Lane CR, Lim EP, Kalyanaraman N, Nemesh J, Ziaugra L, Friedland L, Rolfe A, Warrington J, Lipshutz R, Daley GQ, Lander ES (1999): Characterization of single-nucleotide polymorphisms in coding regions of human genes. Nat Genet 22:231-238

Chee M, Yang R, Hubbell E, Berno A, Huang XC, Stern D, Winkler J, Lockhart DJ, Morris MS, Fodor SP (1996): Accessing genetic information with high-density DNA arrays. Science 274:610-614

Coutts RT (1994): Polymorphism in the metabolism of drugs, including antidepressant drugs: comments on phenotyping. J Psychiatry Neurosci 19:30-44

Dahl ML, Bertilsson L, Nordin C (1996): Steady-state plasma levels of nortriptyline and its 10-hydroxy metabolite: relationship to the CYP2D6 genotype. Psychopharmacology 123:315-319

Dalen P, Dahl ML, Ruiz ML, Nordin J, Bertilsson L (1998): 10-hydroxylation of nortriptyline in white persons with $0,1,2,3$, and 13 functional CYP2D6 genes. Clin Pharmacol Ther 63:444-452

Daly AK, Brockmoller J, Broly F, Eichelbaum M, Evans WE, Gonzalez FJ, Huang JD, Idle JR, Ingelman-Sundberg $M$, Ishizaki T, Jacqz-Aigrain E, Meyer UA, Nebert DW, Steen VM, Wolf CR, Zanger UM (1996): Nomenclature for human CYP2D6 alleles. Pharmacogenetics 6:193-201

Fodor S (1997): Massively parallel genomics. Science 277:393-395

Hirschhorn JN, Sklar P, Lindblad-Toh K, Lim YM, RuizGutierrez M, Bolk S, Langhorst B, Schaffner S, Winchester E, Lander ES (2000): SBE-TAGS: An array-based method for efficient single-nucleotide polymorphism genotyping. Proc Natl Acad Sci USA 97:12164-12169

Lee CK, Klopp RG, Weindruch R, Prolla TA (1999): Gene expression profile of aging and its retardation by caloric restriction. Science 285:1390-1393

Linder MW, Prough RA, Valdes Jr R (1997): Pharmacogenetics: a laboratory tool for optimizing therapeutic efficiency. Clin Chem 43:254-266

Marez D, Legrand M, Sabbagh N, Guidice JM, Spire C, Lafitte JJ, Meyer UA, Broly F (1997): Polymorphism of the cytochrome P450 CYP2D6 gene in a European population: characterization of 48 mutations and 53 alleles, their frequencies and evolution. Pharmacogenetics 7:193-202

Morita S, Shimoda K, Someya T, Yoshimura Y, Kamijima K, Kato N (2000): Steady-state plasma levels of nortriptyline and its hydroxylated metabolites in Japanese patients: impact of CYP2D6 genotype on the hydroxylation of nortriptyline. J Clin Psychopharmacol 20:141-149

Mulsant BH, Foglia JP, Sweet RA, Rosen J, Lo KH, Pollock BG (1997): The effects of perphenazine on the concentration of nortriptyline and its hydroxymetabolites in older patients. J Clin Psychopharmacol 17:318-321

Olesen OV, Linnet K (1997): Hydroxylation and demethylation of the tricyclic antidepressant nortriptyline by cDNA-expressed human cytochrome P-450 isozymes. Drug Metabolism and Disposition 25:740-744

Perry PJ, Zeilmann C, Arndt S (1994): Tricyclic antidepres- 
sant concentrations in plasma: an estimate of their sensitivity and specificity as a predictor of response. J Clin Psychopharmacol 14:230-240

Pollock BG (1999): Adverse reactions of antidepressants in elderly patients. Journal of Clinical Psychiatry 60 Suppl 20:4-8

Pollock BG, Everett G, Perel JM (1992): Comparative cardiotoxicity of nortriptyline and its isomeric 10hydroxymetabolites. Neuropsychopharmacology 6:1-10

Pollock BG, Perel JM, Paradis CF, Fasiczka AL, Reynolds III CF (1994): Metabolic and physiologic consequences of nortriptyline treatment in the elderly. Psychopharmacol Bull 30:145-150

Potter WZ, Manji HK, Rudorfer MV (1998): Tricyclics and tetracyclics. In Schatzberg AF, Nemeroff CB (eds), Textbook of Psychopharmacology, 2nd ed. Washington, DC, American Psychiatric Press, pp 199-218

Reynolds CF, 3rd, Perel JM, Frank E, Cornes C, Miller MD, Houck PR, Mazumdar S, Stack JA, Pollock BG, Dew MA, Kupfer DJ (1999): Three-year outcomes of maintenance nortriptyline treatment in late-life depression: a study of two fixed plasma levels. Am J Psychiatry 156:1177-1181
Robinson RG, Schultz SK, Castillo C, Kopel T, Kosier JT, Newman RM, Curdue K, Petracca G, Starkstein SE (2000): Nortriptyline versus fluoxetine in the treatment of depression and in short-term recovery after stroke: A placebo-controlled, double-blind study. Am J Psychiatry 157:351-359

Sachse C, Brockmoller J, Bauer S, Roots I (1997): Cytochrome P450 2D6 variants in a Caucasian population: allele frequencies and phenotypic consequences. Am J Hum Genet 60:284-295

Steen VM, Andreassen OA, Daly AK, Tefre T, Borresen AL, Idle JR, Gulbrandsen AK (1995): Detection of the poor metabolizer-associated CYP2D6(D) gene deletion allele by long-PCR technology. Pharmacogenetics 5:215-223

Watson SJ, Akil H (1999): Gene chips and arrays revealed: a primer on their power and their uses. Biol Psychiatry 45:533-543

Yue QY, Zhong ZH, Tybring G, Dalen P, Dahl ML, Bertilsson L, Sjoqvist F (1998): Pharmacokinetics of nortriptyline and its 10-hydroxy metabolite in Chinese subjects of different CYP2D6 genotypes. Clin Pharmacol Ther 64:384-390. 